\title{
CHARACTERIZING PROJECTIVE SPACES FOR VARIETIES WITH AT MOST QUOTIENT SINGULARITIES
}

\author{
JIUN-CHENG CHEN
}

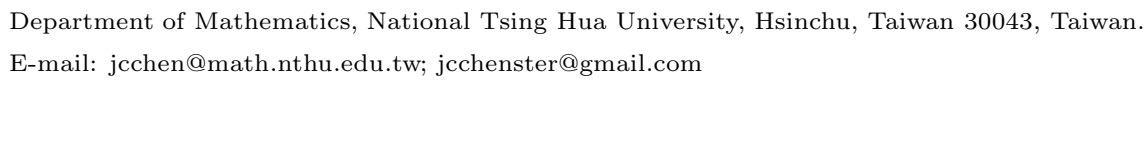

Abstract

We generalize the well-known numerical criterion for projective spaces by Cho, Miyaoka and Shepherd-Barron to varieties with at worst quotient singularities. Let $X$ be a normal projective variety of dimension $n \geq 3$ with at most quotient singularities. Our result asserts that if $C \cdot\left(-K_{X}\right) \geq n+1$ for every curve $C \subset X$, then $X \cong \mathbb{P}^{n}$.

\section{Introduction}

We work over the field $\mathbb{C}$. The $n$-dimensional projective space $\mathbb{P}^{n}$ is probably the simplest compact (projective) complex manifold. Let $K_{\mathbb{P}^{n}}$ be the canonical bundle (the line bundle of holomorphic $n$-forms). It is elementary to see that the $-K_{\mathbb{P}^{n}}=\mathcal{O}(n+1)$. In particular, $($ line $) \cdot\left(-K_{X}\right)=n+1$ and $C \cdot\left(-K_{\mathbb{P}^{n}}\right) \geq n+1$ for all curves $C \subset \mathbb{P}^{n}$. This is a rather unusual property: recall that if $X$ is a smooth projective variety of dimension $n$ and $K_{X}$ is not nef, then the Cone theorem implies that there is a rational curve $C$ such that $0<C \cdot\left(-K_{X}\right) \leq n+1$.

From this perspective, the anti-canonical bundle $-K_{\mathbb{P} n}$ is unusually positive. It turns out that this property characterizes $\mathbb{P}^{n}$ among smooth projective varieties of dimension $n$.

Received September 11, 2017.

AMS Subject Classification: 14D06, 14D23, 14E08, 14J40, 14J17.

Key words and phrases: Projective space, quotient singularity, pseudo-index, deformation theory, twisted stable curves. 
Theorem 1.1 ([4] and [10]). Let $X$ be a smooth projective variety of dimension $n \geq 3$. Assume that $C \cdot\left(-K_{X}\right) \geq n+1$ for all curves $C \subset X$. Then $X \cong \mathbb{P}^{n}$.

This result was first proved by Cho, Miyaoka and Shepherd-Barron [4] and later by Kebekus [10].

Since the condition

$$
C \cdot\left(-K_{X}\right) \geq n+1 \forall C \subset X
$$

is very strong, it is natural to ask if the assumption on smoothness is necessary. In [5], H,-H, Tseng and the author proved the characterization result assuming that $X$ has only isolated LCIQ singularities. The main goal of this paper is to further weaken the smoothness assumption; it builds upon methods developed in [5]. The precise statement of our result is the following:

Theorem 1.2. Let $X$ be a projective variety of dimension $n \geq 3$ with at most quotient singularities. Assume that

$$
C \cdot\left(-K_{X}\right) \geq n+1 \forall C \subset X
$$

Then $X \cong \mathbb{P}^{n}$.

We now explain the strategy used in [10]. First consider the projective space $\mathbb{P}^{n}$. Let $p \in \mathbb{P}^{n}$ be any point. Let $\widetilde{\mathbb{P}^{n}}$ be the blowing up of $\mathbb{P}^{n}$ along the point $p$. The variety $\widetilde{\mathbb{P}^{n}}$ is a $\mathbb{P}^{1}$-bundle over $\mathbb{P}^{n-1}$. Let $E \cong \mathbb{P}^{n-1} \subset \widetilde{\mathbb{P}^{n}}$ be the exceptional divisor. The normal bundle of the exceptional divisor $E \cong \mathbb{P}^{n-1}$ is $\mathcal{O}_{\mathbb{P}^{n-1}}(-1)$. The variety $\widetilde{\mathbb{P}^{n}}$ is the Chow family of minimal degree rational curves (lines) through the point $p \in \mathbb{P}^{n}$.

Now consider the variety $X$. Take a general point $x \in X$. Denote by $\tilde{X}$ the blowing up of $X$ along $x$. If we can prove that $\tilde{X}$ is the Chow family of minimal degree (with respect to an ample line bundle) rational curves through $x$, then we have a good chance to prove that $\tilde{X} \cong \widetilde{\mathbb{P}^{n}}$ and hence $X \cong \mathbb{P}^{n}$.

When $X$ is smooth, Kebekus proved that the Chow scheme $H_{x}$ of minimal degree rational curves through a general point $x \in X$ is isomorphic to $\mathbb{P}^{n-1}$ and $\tilde{X} \cong \widetilde{\mathbb{P}^{n}}\left[10\right.$. It follows easily that $X \cong \mathbb{P}^{n}$. One important step 
in his proof is to show that $X$ has a lot of minimal degree rational curves through $x$. More precisely, one needs to show that

$$
\operatorname{dim} H_{x} \geq l \cdot\left(-K_{X}\right)-2=n-1
$$

where $[l] \in H_{x}$. Note that $\operatorname{dim} H_{x} \leq n-1$ by a standard bend and break argument. It follows that the Chow scheme $H_{x}$ has the expected dimension $n-1$. Kebekus then proved that the tangent map $H_{x} \rightarrow \mathbb{P}^{n-1}$ is an isomorphism [9] [10].

When $X$ is possibly singular, the situation is quite different: it is difficult to have the desired lower bound on the dimension of $H_{x}$. In [5] (joint work with H.-H. Tseng), we proved the characterization result when $X$ has at worst isolated LCIQ singularities. We used the Deligne-Mumford stack $\mathcal{X} \rightarrow$ $X$, twisted stable maps into $\mathcal{X}$ and Mori's bend and break techniques to show the existence of a rational curve $f: \mathbb{P}^{1} \rightarrow X$ such that $(1) f\left(\mathbb{P}^{1}\right) \cdot\left(-K_{X}\right)=$ $n+1$, and $(2) f\left(\mathbb{P}^{1}\right)$ does not meet the singular locus of $X$. Using this fact, one can show (following [10]) that $H_{x} \cong \mathbb{P}^{n-1}$ and $X \cong \mathbb{P}^{n}$. In [5], the condition that $X_{\text {sing }}$ is isolated is essential; it ensures that we can deform a specific rational curve in $X$. Our methods do not apply without this assumption.

In this paper, we develop methods to study the case when $X$ has only quotient singularities (not necessarily isolated). The main idea is quite simple: instead of considering the twisted stable map directly, we consider a double cover and study the possible degeneration types. The assumption

$$
C \cdot\left(-K_{X}\right) \geq n+1 \forall C \subset X
$$

ensures that the possible degeneration types are limited and we are able to show the existence of a minimal degree rational curve $f: \mathbb{P}^{1} \rightarrow X$ which does not meet $X_{\text {sing }}$. Once this fact is established, it is standard [10] [5] to prove that the Chow family has the right dimension, i.e. $n-1$, and $X \cong \mathbb{P}^{n}$.

A few words on using Deligne-Mumford stacks: for a singular variety $X$, it is difficult to prove the existence of enough rational curves via deformation theory; obtaining a lower bound of the dimension $\operatorname{Mor}\left(\mathbb{P}^{1}, X\right)$ (in terms of $-K_{X}$-degree) is difficult. However, if $X$ has only local complete intersection quotient (LCIQ) singularities, one can study representable morphisms from a 
twisted curve $\|$ to the stack $\mathcal{X}$ whose coarse moduli space is $X$. This provides a reasonable alternative. We can obtain a lower bound on the dimension of the space of twisted stable maps expressed in terms of the $-K_{X}$-degree and the number of twisted points, see [6].

The rest of this paper is organized as follows: In Section 2, we recall basic definitions on twisted curves and twisted stable maps. We also give a formula on the lower bound on the morphism space. The main proposition (Proposition 3.7) is proved in Section 3. In Section 4, we conclude the proof of the main theorem. We also make a few remarks in that section.

\section{Acknowledgments}

Part of this research was conducted while the author was attending the JAMI conference at Johns Hopkins University. He likes to thank the conference organizers. He also likes to thank Dan Abramovich, Lawrence Ein and Stefan Kebekus for helpful discussions and valuable suggestions. He is partially supported by the Gold Jade Fellowship from Kenda Foundation and National Science Council, Taiwan.

\section{Twisted Curves and Twisted Stable Maps}

Twisted curves play an important role in this paper. One of the main motivations of introducing twisted curves is to compactify the space (DeligneMumford stack) of stable maps into a proper Deligne-Mumford stack [2]. Roughly speaking, twisted curves are nodal curves having certain stack structures étale locally near nodes (and, for pointed curves, marked points). For the precise definition, see [2], Definition 4.1.2.

Let $\mathcal{C}$ be a twisted curve and $C$ its coarse moduli space.

\subsubsection{Nodes}

For a positive integer $r$, let $\mu_{r}$ denote the cyclic group of $r$-th roots of unity. Étale locally near a node, a twisted curve $\mathcal{C}$ is isomorphic to the stack

\footnotetext{
${ }^{1}$ Roughly speaking, this is a one dimensional Deligne-Mumford stack with isolated cyclic quotient stack structures and with coarse moduli space being a nodal curve [2].
} 
quotient $\left[U / \mu_{r}\right]$ of the nodal curve $U=\{x y=f(t)\}$ by the following action of $\mu_{r}$ :

$$
(x, y) \mapsto\left(\zeta_{r} x, \zeta_{r}^{-1} y\right)
$$

where $\zeta_{r}$ is a primitive $r$-th root of unity. Étale locally near this node, the coarse curve $C$ is isomorphic to the schematic quotient $U / \mu_{r}$.

\subsubsection{Markings}

Étale locally near a marked point, $\mathcal{C}$ is isomorphic to the stack quotient $\left[U / \mu_{r}\right]$. Here $U$ is a smooth curve with local coordinate $z$ defining the marked point, and the $\mu_{r}$-action is defined by

$$
z \mapsto \zeta_{r} z
$$

Near this marked point the coarse curve is the schematic quotient $U / \mu_{r}$.

\subsection{Twisted stable maps}

Definition 2.1. A twisted $n$-pointed stable map of genus $g$ and degree $d$ over a scheme $S$ consists of the following data (see [2], Definition 4.3.1):

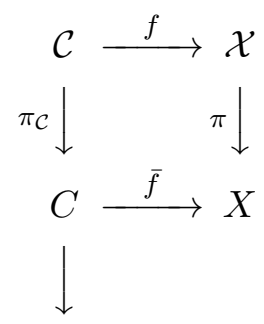

$S$.

along with $n$ closed substacks $\Sigma_{i} \subset \mathcal{C}$ such that

(1) $\mathcal{C}$ is a twisted nodal $n$-pointed curve over $S$ (see [2], Definition 4.1.2),

(2) $f: \mathcal{C} \rightarrow \mathcal{X}$ is representable,

(3) $\Sigma_{i}$ is an étale gerbe over $S$, for $i=1, \ldots, n$, and

(4) the map $\bar{f}:\left(C,\left\{p_{i}\right\}\right) \rightarrow X$ between coarse moduli spaces induced from $f$ is a stable $n$-pointed map of degree $d$ in the usual sense. 
A twisted map $f: \mathcal{C} \rightarrow \mathcal{X}$ is stable if and only if for every irreducible component $\mathcal{C}_{i} \subset \mathcal{C}$, one of the following cases holds:

(1) $\left.f\right|_{\mathcal{C}_{i}}$ is nonconstant,

(2) $\left.f\right|_{\mathcal{C}_{i}}$ is constant, and $\mathcal{C}_{i}$ is of genus at least 2 ,

(3) $\left.f\right|_{\mathcal{C}_{i}}$ is constant, $\mathcal{C}_{i}$ is of genus 1 , and there is at least one special points on $\mathcal{C}_{i}$,

(4) $\left.f\right|_{\mathcal{C}_{i}}$ is constant, $\mathcal{C}_{i}$ is of genus 0 , and there are at least three special points on $\mathcal{C}_{i}$.

In particular, a nonconstant representable morphism from a smooth twisted curve to $\mathcal{X}$ is stable.

We say a twisted stable map $\mathcal{C} \rightarrow \mathcal{X}$ is rational if the coarse moduli space $C$ of $\mathcal{C}$ is rational.

Let $\mathcal{K}_{g, n}(\mathcal{X}, d)$ denote the category of twisted $n$-pointed stable maps to $\mathcal{X}$ of genus $g$ and degree $d$. The main result of [2] is that $\mathcal{K}_{g, n}(\mathcal{X}, d)$ is a proper Deligne-Mumford stack with projective coarse moduli space denoted by $K_{g, n}(\mathcal{X}, d)$. Let $\beta \in H_{2}(X)$ be a homology class. The space of twisted $n$ pointed stable maps $f: \mathcal{C} \rightarrow \mathcal{X}$ of genus $g$ and homology class $\left[(\pi \circ f)_{*}(\mathcal{C})\right]=$ $\beta$ is denoted by $\mathcal{K}_{g, n}(\mathcal{X}, \beta)$. This stack is also proper [2].

\subsection{Morphism space from a twisted curve to a Deligne-Mumfors stack}

In this paper, we use both the stack of twisted stable maps and the morphism space from $\mathcal{C}$ to $\mathcal{X}$. Roughly speaking, an element in the morphism space $\operatorname{Mor}(\mathcal{C}, \mathcal{X})$ is a twisted stable map together with a parameterization on the source curve $\mathcal{C}$. Let $\Sigma \subset \mathcal{C}$ be the set of twisted points and $B \subset \mathcal{C}$ a finite set of points (twisted or untwisted). Let $f: \mathcal{C} \rightarrow \mathcal{X}$ be a representable morphism. When $\mathcal{X}$ is smooth, we have a lower bound on the dimension of $\operatorname{Mor}\left(\mathcal{C}, \mathcal{X} ;\left.f\right|_{B}\right)$ near the morphism $[f]$.

Lemma $2.2(=6]$ Lemma 4.4).

$\operatorname{dim}_{[f]} \operatorname{Mor}\left(\mathcal{C}, \mathcal{X} ;\left.f\right|_{B}\right) \geq-\mathcal{C} \cdot K_{\mathcal{X}}+n\left[\chi\left(\mathcal{O}_{\mathcal{C}}\right)-\operatorname{Card}(B)\right]-\sum_{x \in \Sigma \backslash B} \operatorname{age}\left(f^{*} T \mathcal{X}, x\right)$ 
Remark 2.3. The age term contribution from each twisted point can be computed using local stack structure of that twisted point. For each twisted point, the age term is strictly less than $\operatorname{dim} X$.

Remark 2.4. When $\mathcal{X}$ has only LCI singularities, a similar formula still holds as long as the image of $\mathcal{C}$ does not lie completely in $\mathcal{X}_{\text {sing }}$ (the locus where the stack $\mathcal{X}$ is singular).

\subsection{Lifting}

Let $X$ be a normal projective variety with quotient singularities and $\mathcal{X}$ a proper smooth Deligne-Mumford stack such that $\pi: \mathcal{X} \rightarrow X$ is isomorphic over $X_{\text {reg }}=X-X_{\text {sing }}$ and $X$ is a coarse moduli space of $\mathcal{X}$. Let $C$ be a smooth irreducible curve and $\bar{f}: C \rightarrow X$ a morphism.

We want to "lift" the map $\bar{f}: C \rightarrow X$ to a map $C \rightarrow \mathcal{X}$. In general, this is not possible unless we endow a orbicurve structure on $C$ [2] Lemma 7.2.5, or pass to a finite cover $C^{\prime} \rightarrow C$ [2] Theorem 7.1.1.

First consider the case when the image $\bar{f}(C)$ meets the smooth locus of $X$. Let $\left\{p_{i} \mid i \in I\right\} \subset C$ be the finite set of points which are mapped to the singular locus of $X$, and let $C_{0}=C \backslash\left\{p_{i} \mid i \in I\right\}$. Since $X$ is isomorphic to $\mathcal{X}$ away from the singular locus $X_{\text {sing }}$, the map $\left.\bar{f}\right|_{C_{0}}: C_{0} \rightarrow X$ admits a lifting $C_{0} \rightarrow \mathcal{X}$. By [2], Lemma 7.2.5, there exists a twisted curve $\mathcal{C}$ with coarse moduli space $C$, and a twisted stable map $f: \mathcal{C} \rightarrow \mathcal{X}$ extending $C_{0} \rightarrow \mathcal{X}$.

Now consider the case when $\bar{f}(C) \subset X_{\text {sing }}$. Let $\eta \in C$ be the generic point of $C$. Consider the morphism $\eta \in C \rightarrow X$. After a finite extension $\eta^{\prime} \rightarrow \eta$, there is a lifting $\eta^{\prime} \rightarrow \mathcal{X}$ since $\mathcal{X}$ is proper. Let $\eta^{\prime} \in C^{\prime}$ be the smooth irreducible curve. Note that the morphism $\eta^{\prime} \rightarrow \mathcal{X}$ is defined over an open set $U \rightarrow \mathcal{X}$. One can extend the finite morphism $\eta^{\prime} \rightarrow \eta$ to $C^{\prime} \rightarrow C$ since $C$ is proper. Let $C^{\prime}-U=\left\{p_{j} \mid j \in J\right\}$. Endowing stack structures on this finite set of points, we can extend the morphism $U \rightarrow \mathcal{X}$ to $\mathcal{C}^{\prime} \rightarrow \mathcal{X}$. After a further finite cover $C^{\prime \prime} \rightarrow C^{\prime}$, we can find a morphism $C^{\prime \prime} \rightarrow \mathcal{X}$. 


\section{Untwisted Rational Curves}

In this section, we prove the existence of "good" rational curves with $-K_{X}$-degree $n+1$, i.e. rational curves which do not intersect the singular locus of $X$.

Consider a smooth Fano variety $X$. Let $x \in X$ be a general point and $S=\left\{x_{1}, x_{2}, \cdots, x_{k}\right\} \subset X$ be any finite set of points which does not contain $x$. It is possible to find a rational curve through the point $x$ which misses the finite set $S=\left\{x_{1}, x_{2}, \cdots, x_{k}\right\} \subset X$. This property is, however, not true for singular varieties.

Example 3.1. Let $E \subset \mathbb{P}^{2}$ be a smooth elliptic curve. Let $X \subset \mathbb{P}^{3}$ be the projective cone of $E$. The surface $X$ has only one LCI singularity at the vertex. The surface $X$ is Fano and all rational curves pass through the vertex.

This example shows that the existence of a rational curve which does not meet the singular locus $X_{\text {sing }}$ is non-trivial.

Notation 3.2. Let $X$ be a normal projective variety with at worst quotient singularities. Fix a proper smooth Deligne-Mumford stack $\pi: \mathcal{X} \rightarrow X$ such that $X$ is a coarse moduli space of $\mathcal{X}$ and $\pi$ is an isomorphism over $X_{\text {reg }}=X \backslash X_{\text {sing }}$. Note that $K_{\mathcal{X}}=\pi^{*} K_{X}$ and

$$
\mathcal{C} \cdot K_{\mathcal{X}}=C \cdot K_{X}
$$

for any (twisted) curve $\mathcal{C} \rightarrow \mathcal{X}$ with coarse curve $C[6]$.

We start with the following lemma:

Lemma 3.3. Let $R \subset \overline{N E}(X)$ be any $K_{X}$-negative extremal ray. Then there exists a twisted rational curve $f: \mathcal{C} \rightarrow \mathcal{X}$ such that $(1) \mathcal{C}$ has at most one twisted point, (2) the intersection number $\mathcal{C} \cdot\left(-K_{\mathcal{X}}\right) \leq n+1$, and (3) $\left[(\pi \circ f)_{*}(\mathcal{C})\right] \in R$.

Proof. This is essentially Proposition 3.1 in [5]; one only needs to note that the argument goes through when the stack $\mathcal{X}$ has only isolated LCI singularities.

Unless mentioned otherwise, we make further assumptions in the rest of this paper: 
Assumptions 3.4. From now on we assume that $X$ is a projective variety of $\operatorname{dim} X=n \geq 3$ with at most quotient singularities and has the property that

$$
C \cdot\left(-K_{X}\right) \geq n+1
$$

for every curve $C \subset X$.

Lemma 3.5. Assumptions as in Assumptions 3.4. Let $R \in \overline{N E}(X)$ be any $K_{X}$-negative extremal ray and $x \in \mathcal{X}$ a general point. Then there exists a twisted rational curve $f: \mathcal{C} \rightarrow \mathcal{X}$ such that (1) $\mathcal{C}$ has at most 1 twisted point, (2) $x \in f(\mathcal{C})$, (3) $\left[(\pi \circ f)_{*}(\mathcal{C})\right] \in R \subset H_{2}(X)$, and (4) $\mathcal{C} \cdot f^{*}\left(-K_{\mathcal{X}}\right)=n+1$.

Proof. We only need to verify the condition (2) by Lemma 3.3. Let $f: \mathcal{C} \rightarrow$ $\mathcal{X}$ be a twisted rational curve as in Lemma 3.3 and $\infty \in \mathcal{C}$ the twisted point on $\mathcal{C}$. By Lemma 2.2.

$$
\operatorname{dim}_{[f]} \operatorname{Mor}\left(\mathcal{C}, \mathcal{X},\left.f\right|_{\infty}, n+1\right) \geq(n+1)+(1-1) n=n+1 .
$$

Denote by $\mathcal{H}_{f(\infty)} \subset \mathcal{K}_{0,1}\left(\mathcal{X},\left[(\pi \circ f)_{*} \mathcal{C}\right]\right)$ the stack of twisted stable maps through the twisted point $f(\infty)$.

Abusing the notation, we also view $[f] \in \operatorname{Mor}\left(\mathcal{C}, \mathcal{X},\left.f\right|_{\infty}, n+1\right)$ as an element of $\mathcal{H}_{f(\infty)}$. Note that $\operatorname{dim}_{[f]} \mathcal{H}_{f(\infty)} \geq n-1$.

Let $T \rightarrow \mathcal{H}_{f(\infty)}$ be any quasi-finite morphism where $T$ is a normal irreducible quasi-projective variety of dimension $n-1$ such that $[f] \in \operatorname{Im}(T)$. Consider the pull back family $U_{T} \rightarrow T$ of twisted stable maps and the morphism $i_{T}: U_{T} \rightarrow \mathcal{X}$. Forgetting the stack structures on the source curves and on the target stack $\mathcal{X}$, one obtains a family of stable maps (into $X)$. Denote this family of stable maps by $\phi_{T}: \bar{U}_{T} \rightarrow T$ and the morphism (into $X$ ) by $\bar{i}_{T}: \bar{U}_{T} \rightarrow X$.

Note that the homology class $\left[(\pi \circ f)_{*}(\mathcal{C})\right]$ can not be written as the sum of at least two curve classes by Assumptions 3.4. Let $q=(\pi \circ f)(\infty)$.

Recall that $\mathcal{K}_{0,1}\left(\mathcal{X},\left[(\pi \circ f)_{*}(\mathcal{C})\right]\right) \rightarrow \mathcal{K}_{0,1}\left(X,\left[(\pi \circ f)_{*}(\mathcal{C})\right]\right)$ is quasi-finite 2]. Being the composition of two quasi-finite morphisms, the morphism $T \rightarrow \mathcal{H}_{f(\infty)} \rightarrow \mathcal{K}_{0,1}\left(X,\left[(\pi \circ f)_{*}(\mathcal{C})\right]\right)$ is also quasi-finite.

Claim 3.6. The morphism $\bar{i}_{T}: \bar{U}_{T} \rightarrow X$ is quasi-finite away from the preimage of $q$. 
Proof. This is a standard bend and break argument. Note that every fiber of the family $\phi_{T}: \bar{U}_{T} \rightarrow T$ is irreducible since the homology class $\left[(\pi \circ f)_{*} \mathcal{C}\right]$ is unbreakable. It follows that no fiber of $\phi_{T}: \bar{U}_{T} \rightarrow T$ is contracted by the morphism $\bar{i}_{T}$.

Suppose that $\bar{i}_{T}$ is not quasi-finite (away from the preimage of $q$ ). Then there is a curve $C \subset \bar{U}_{T}$ (may not be projective) such that $\bar{i}_{T}(C)=q_{1} \neq q$. Let $\phi_{T}(C) \subset T$ be the image of $C$ and $C^{\prime} \rightarrow \phi_{T}(C)$ the normalization. Note that the morphism

$$
C^{\prime} \rightarrow \phi_{T}(C) \subset T \rightarrow \mathcal{K}_{0,1}\left(X,\left[(\pi \circ f)_{*}(\mathcal{C})\right]\right)
$$

is still quasi-finite. Pull back the family of stable maps to the curve $C^{\prime}$. Compactify this family of stable maps; this is a family (over a proper curve $\bar{C}^{\prime}$ ) of stable maps whose image contains $q$ and $q_{1}$. This contradicts the unbreakable assumption on the homology class $\left[(\pi \circ f)_{*}(\mathcal{C})\right]$ by Mori's bend and break.

By Claim 3.6, it follows that the dimension of the image $\bar{i}_{T}\left(\bar{U}_{T}\right)$ (and the dimension of $\left.\left(\pi \circ i_{T}\right)\left(U_{T}\right)\right)$ is $n$. Thus a general point $x \in \mathcal{X}$ lies on the image of a twisted stable map which satisfies the conditions (1), (3), and (4). This concludes the proof.

Proposition 3.7. Assumptions as in Assumptions 3.4. Let $R \subset \overline{N E}(X)$ be any $K_{X}$-negative extremal ray. There is a rational curve $f: \mathbb{P}^{1} \rightarrow \mathcal{X}$ such that (1) the image $\pi \circ f\left(\mathbb{P}^{1}\right)$ does not lie in $X_{\text {sing }},(2) x \in f\left(\mathbb{P}^{1}\right)$ for a general point $x \in \mathcal{X}$, (3) the class $\left[\pi \circ f\left(\mathbb{P}^{1}\right)\right] \in R$, and $(4) f^{*} \mathbb{P}^{1} \cdot\left(-K_{\mathcal{X}}\right)=n+1$.

Proof. By Lemma 3.5, there is a twisted rational curve $\mathcal{C} \rightarrow \mathcal{X}$ such that (1) $\mathcal{C}$ has at most one twisted point, $(2) x_{0} \in f(\mathcal{C})\left(x_{0}\right.$ is a general point of $\mathcal{X}),(3)\left[(\pi \circ f)_{*}(\mathcal{C})\right] \in R \subset H_{2}(X)$, and $(4) \mathcal{C} \cdot f^{*}\left(-K_{X}\right)=n+1$.

Let $\beta=\left[(\pi \circ f)_{*} \mathcal{C}\right] \in H_{2}(X)$. We fix this homology class in the rest of the proof.

We may assume that $\mathcal{C}$ does have a twisted point, denoted by $\infty \in \mathcal{C}$. Denote by $x_{\infty}=f(\infty)$ the image of $\infty$. May assume that $f(0)=x_{0}$ and $x_{0} \in \pi^{-1}\left(X_{r e g}\right)$. 
Step 0. Double cover of $\mathcal{C}$ :

Recall that the coarse curve of $\mathcal{C}$ is $\mathbb{P}^{1}$. Choose a 2-to-1 cover $h: \mathbb{P}^{1} \rightarrow \mathbb{P}^{1}$ such that $h(0)=0, \quad h(\infty)=\infty$ and $h$ is ramified at $\{0, \infty\} \subset \mathbb{P}^{1}$, e. g. $h(z)=z^{2}$. Choosing a suitable stack structure at $\infty \in \mathbb{P}^{1}$, we can lift $h: \mathbb{P}^{1} \rightarrow \mathbb{P}^{1}$ to $\mathcal{D} \rightarrow \mathcal{C}$ where $\mathcal{D}$ is a twisted rational curve. We abuse the notation and still denote the morphism on the stack level by $h: \mathcal{D} \rightarrow \mathcal{C}$. Denote the stacky point on $\mathcal{D}$ by $\infty$ and the preimage of $0 \in \mathcal{C}$ on $\mathcal{D}$ by 0 (an untwisted point).

The composition $f \circ h: \mathcal{D} \rightarrow \mathcal{X}$ is a twisted stable map into $\mathcal{X}$ with $-K_{\mathcal{X}}$ degree $2 n+2$. Note that the homology class $\left[(\pi \circ f \circ h)_{*}(\mathcal{D})\right]=2 \beta \in H_{2}(X)$.

Step 1. Bend and break:

We first show that we can deform the curve $f \circ h: \mathcal{D} \rightarrow \mathcal{X}$. Let $\operatorname{Mor}(\mathcal{D}, \mathcal{C}, 2)$ be the space of of degree 2 representable morphisms from $\mathcal{D}$ to $\mathcal{C}$. Let $\operatorname{Mor}\left(\mathcal{D}, \mathcal{C},\left.h\right|_{\{0, \infty\}}, 2\right) \subset \operatorname{Mor}(\mathcal{D}, \mathcal{C}, 2)$ be the subspace consisted of morphisms $h_{1}: \mathcal{D} \rightarrow \mathcal{C}$ such that $h_{1}(0)=0$ and $h_{1}(\infty)=\infty$. The space $\operatorname{Mor}\left(\mathcal{D}, \mathcal{C},\left.h\right|_{\{0, \infty\}}, 2\right)$ has dimension 3 .

By Lemma 2.2,

$$
\operatorname{dim}_{[f \circ h]} \operatorname{Mor}\left(\mathcal{C}, \mathcal{X},\left.(f \circ h)\right|_{\{0, \infty\}}, 2 \beta\right) \geq 2 n+2+(1-2) n=n+2>3 .
$$

Thus we can deform the morphism $f \circ h: \mathcal{D} \rightarrow \mathcal{X}$ such that the image deforms in $\mathcal{X}$. This also implies that there is a morphism $\left[f^{\prime}\right] \in \operatorname{Mor}(\mathcal{D}, \mathcal{X}, 2 \beta)$ such that $f^{\prime}: \mathcal{D} \rightarrow \mathcal{X}$ is birational to its image.

Choose $[g] \in \operatorname{Mor}(\mathcal{D}, \mathcal{X}, 2 \beta)$ such that $g: \mathcal{D} \rightarrow \mathcal{X}$ is birational to its image in $\mathcal{X}$ and $g(0)=x_{0}$. Let $1 \in \mathcal{D}$ be an untwisted point and $x_{1}=g(1)$ its image in $\mathcal{X}$.

Claim 3.8. Replacing $[g] \in \operatorname{Mor}(\mathcal{D}, \mathcal{X}, 2 \beta)$ and the point $x_{1}$ if necessary, we may assume that $x_{1} \in \pi^{-1}\left(X_{\text {reg }}\right), x_{1} \neq x_{0}$, and $x_{1}$ does not lie on the image of $[h]$ where $[h] \in \mathcal{K}_{0, k}(\mathcal{X}, \beta), k=1,2$ is any twisted stable map through $x_{0}$ and $x_{\infty}$.

Proof. Let $\mathcal{K}_{1} \subset \mathcal{K}_{0,1}(\mathcal{X}, 2 \beta)$ be the family of twisted stable maps whose image contains $x_{0}$ and $x_{\infty}$. View the morphism $g: \mathcal{D} \rightarrow \mathcal{X}$ as an element of 
$\mathcal{K}_{1}$. Note that

$$
\begin{gathered}
\operatorname{dim}_{[g]} \mathcal{K}_{1}=\operatorname{dim} \operatorname{Mor}_{[g]}\left(\mathcal{D}, \mathcal{X},\left.g\right|_{\{0, \infty\}}, 2 \beta\right)-\operatorname{dim} \operatorname{Aut}(\mathcal{D},\{0, \infty\}) \\
\geq 2 n+2-(1-2) n-1=n+1
\end{gathered}
$$

Here we use Lemma 2.2 and the fact $\operatorname{dim} \operatorname{Aut}(\mathcal{C},\{0, \infty\})=1$. The image of this family is at least a surface since the image of $g: \mathcal{D} \rightarrow \mathcal{X}$ deforms.

Consider all possible twisted stable maps $[h] \in \mathcal{K}_{0, k}(\mathcal{X}, \beta), k=1,2$ such that the points $x_{0}$ and $x_{\infty}$ lie on the image of $h$. By Lemma 3.9, there are only finitely many such such twisted stable maps. The image of all these twisted stable maps is one dimensional (in $\mathcal{X}$ ). The claim follows by choosing a suitable $[g] \in \mathcal{K}_{1}$.

Let $\mathcal{K} \subset \mathcal{K}_{0,1}(\mathcal{X}, 2 \beta)$ be the substack of twisted stable maps whose image contains $x_{0}, x_{1}$ and $x_{\infty}$. Compute the dimension of the stack $\mathcal{K} \subset$ $\mathcal{K}_{0,1}(\mathcal{X}, 2 \beta)$ at $[g]:$

$$
\begin{gathered}
\operatorname{dim}_{[g]} \mathcal{K}=\operatorname{dim} \operatorname{Mor}_{[g]}\left(\mathcal{D}, \mathcal{X},\left.g\right|_{\{0,1, \infty\}}, 2 \beta\right)-\operatorname{dim} \operatorname{Aut}(\mathcal{D},\{0,1, \infty\}) \\
\geq 2 n+2-(1-3) n-0=2 .
\end{gathered}
$$

By Mori's bend and break, the domain curve of some twisted stable map $[h] \in \mathcal{K}$ has to degenerate to at least two irreducible components.

Step 2. Analysis on possible types of degenerations:

Since $\mathcal{D} \cdot\left(-K_{\mathcal{X}}\right)=2 n+2$ and every curve has $-K_{\mathcal{X}}$-degree at least $n+1$, only two components of the domain curve are not contracted. Note that the coarse space of domain curve is a rational tree with an extra special point (coming from the original stacky point of $\mathcal{D}$ ). It is easy to see that the domain curve can only break into two or three pieces.

Case I. The domain curve has three irreducible components.

Denote these components by $\mathcal{D}_{1}, \mathcal{D}_{2}$ and $\mathcal{D}_{3}$. Denote by $f_{1}: \mathcal{D}_{1} \rightarrow \mathcal{X}$, $f_{2}: \mathcal{D}_{2} \rightarrow \mathcal{X}$ and $f_{3}: \mathcal{D}_{3} \rightarrow \mathcal{X}$ the twisted stable maps. Let $\mathcal{D}_{2}$ be the component which intersects other two components. Note that $D_{2}$ has to be contracted. 
Note that $\left[\pi \circ f_{1}\left(\mathcal{D}_{1}\right)\right]+\left[\pi \circ f_{3}\left(\mathcal{D}_{3}\right)\right]=\left[\pi \circ g\left(\mathcal{D}_{1}\right)\right]=2 \beta$, and

$$
\left(\pi \circ f_{1}\right)_{*} \mathcal{D}_{1} \cdot\left(-K_{X}\right)=n+1=\left(\pi \circ f_{3}\right)_{*} \mathcal{D}_{3} \cdot\left(-K_{X}\right) .
$$

Since $\beta \in R$ and $R$ is an extremal ray, the class $\left[\pi \circ f_{1}\left(\mathcal{D}_{1}\right)\right]$ is a multiple of the class $\left[\pi \circ f_{3}\left(\mathcal{D}_{3}\right)\right]$. Since they have the same $-K_{X}$-degree, $\left[\pi \circ f_{1}\left(\mathcal{D}_{1}\right)\right]=$ $\left[\pi \circ f_{3}\left(\mathcal{D}_{3}\right)\right]=\beta$.

By symmetry, we only need to consider the following cases:

Case I-a: The stacky point $\infty \in \mathcal{D}_{2}$ and $x_{0} \in f_{1}\left(\mathcal{D}_{1}\right)$ and $x_{1} \in f_{3}\left(D_{3}\right)$.

Case I-b: The stacky point $\infty \in \mathcal{D}_{2}$ and $\left\{x_{0}, x_{1}\right\} \subset f_{1}\left(\mathcal{D}_{1}\right)$.

Case I-a. We will show that Case I-a forms a finite set. Pushing forward to $X$, and noting that

(1) $\pi\left(x_{0}\right) \in\left(\pi \circ f_{1}\right)\left(\mathcal{D}_{1}\right), \quad \pi\left(x_{\infty}\right) \in\left(\pi \circ f_{1}\right)\left(\mathcal{D}_{1}\right)$, and

(2) $\pi\left(x_{1}\right) \in\left(\pi \circ f_{3}\right)\left(\mathcal{D}_{3}\right), \pi\left(x_{\infty}\right) \in \pi \circ f_{3}\left(\mathcal{D}_{3}\right)$,

it follows that there are only finitely many such stable maps by Lemma 3.9.

Case I-b. This is impossible by our choice of $x_{0}, x_{1}$, and $x_{\infty}$ (see Claim 3.8).

Case II. The domain curve has two components, denoted by $\mathcal{D}_{1}$ and $\mathcal{D}_{2}$, intersecting at a node, denoted by $q$.

Consider the following two cases:

Case II-a: the node $q$ is an untwisted point, i.e. $q \in \pi^{-1}\left(X_{r e g}\right)$.

Case II-b: the node $q$ is a twisted point.

Case II-a. This is easy; one of the irreducible components has no twisted point on it.

We divide Case II-b into several subcases. It suffices to study the following subcases by symmetry: 
Case II-b-1: the twisted point $\infty \in \mathcal{D}_{1}$ and $\left\{x_{0}, x_{1}\right\} \subset f_{1}\left(\mathcal{D}_{1}\right)$.

Case II-b-2: the twisted point $\infty \in \mathcal{D}_{2}$ and $\left\{x_{0}, x_{1}\right\} \subset f_{1}\left(\mathcal{D}_{1}\right)$.

Case II-b-3: the twisted point $\infty \in \mathcal{D}_{1}, x_{0} \in f_{1}\left(\mathcal{D}_{1}\right)$ and $x_{1} \in f_{2}\left(\mathcal{D}_{2}\right)$.

Case II-b-1. This subcase is not possible by our choice of the points $x_{1}$, $x_{0}$ and $x_{\infty}$.

Case II-b-2. In this case, $\mathcal{D}_{1}$ has one twisted point and $\mathcal{D}_{2}$ has two twisted points. Consider $f_{1}: \mathcal{D}_{1} \rightarrow \mathcal{X}$. Note that $p=f_{1}(q) \in \mathcal{X}$, the image of the node $q$, is a stacky point on $\mathcal{X}$.

Since the image of $\mathcal{D}_{1}$ contains $x_{1}$ and $x_{0}$ and the class $\beta$ is unbreakable, there are only finitely many such twisted stable maps thanks to Lemma 3.9 . Since every such twisted stable map has only finitely many twisted points (the image only intersects the stacky locus at finitely many points), it follows that there are only finitely many such stacky points $p$ (since $p$ is the image of a twisted point on the source curve).

Recall that $\mathcal{D}_{2}$ has two stacky points, denoted by $\{q, \infty\}$, such that $\left(\pi \circ f_{2}\right)(q)=\pi(p)$ and $\left(\pi \circ f_{2}\right)(\infty)=\pi\left(x_{\infty}\right)$. Consider the morphism $f_{2}: \mathcal{D}_{2} \rightarrow \mathcal{X}$ as an element of $\mathcal{K}_{0,2}(\mathcal{X}, \beta)$. Consider the set of all possible twisted stable maps $[f] \in \mathcal{K}_{0,2}(\mathcal{X}, \beta)$ such that $\pi \circ f(q)=\pi(p)$ and $\pi \circ f(\infty)=\pi\left(x_{\infty}\right)$. This set is a finite set by Lemma 3.10. Since (1) $p$ (the image of the node) is chosen from a finite set, and (2) for every $p$ there are only finitely many such twisted stable maps $[f] \in \mathcal{K}_{0,2}(\mathcal{X}, \beta)$, it follows that there are only finitely many such twisted stable maps $f_{2}: \mathcal{D}_{2} \rightarrow \mathcal{X}$. This shows that Case II-b-2 also forms a finite set.

Case II-b-3.

The image of $f_{1}: \mathcal{D}_{1} \rightarrow \mathcal{X}$ contains $x_{\infty}$ and $x_{0}$. There are only finitely many such $f_{1}: D_{1} \rightarrow \mathcal{X}$ by Lemma 3.9. It follows that the set of all possible nodes $p$ is also finite as in Case II-b-2.

The image of $f_{2}: \mathcal{D}_{2} \rightarrow \mathcal{X}$ contains $p$ and $x_{1}$. By Lemma 3.9 again, the set of all such $f_{2}: \mathcal{D}_{2} \rightarrow \mathcal{X}$ is also finite.

Step III. Concluding the proof: 
By Step II, all bad cases, i.e. Case I-a, Case I-b, Case II-b-1, Case IIb-2 and Case II-b-3, form a finite set. Denote this finite set by $S$. Since $\operatorname{dim} \mathcal{K} \geq 2$, we can find a proper irreducible curve $T \rightarrow \mathcal{K}$ which is finite to its image and does not meet the finite set $S$. Pull back the family over $\mathcal{K}$ to $T$. It follows that Case II-a is the only possible type of degeneration in this family. This concludes the proof.

The next two lemmas are needed in the proof of Proposition 3.7

Lemma 3.9. Let $\beta \in H_{2}(X)$ be a homology class such that $\beta$ is unbreakable, i.e. it can not be written as the sum of at least two (effective) curve classes. Let $x_{1}$ and $x_{2}$ be two distinct points on $\mathcal{X}$ and $x_{1} \in \pi^{-1}\left(X_{\text {reg }}\right)$. Let $k=1,2$. Then there are only finitely many stable maps $[h] \in \mathcal{K}_{0, k}(\mathcal{X}, \beta)$ such that $x_{1} \in h(\mathcal{C})$ and $x_{2} \in h(\mathcal{C})$.

Proof. The proofs of $k=1$ case and $k=2$ case are similar; we only prove $k=1$ case here. For any $[f] \in \mathcal{K}_{0,0}(X, \beta)$, the domain curve is irreducible since $\beta$ is unbreakable. By bend and break, there are only finitely many stable maps $\left[f_{i}\right] \in \mathcal{K}_{0,0}(X, \beta)$ whose image contains $\pi\left(x_{1}\right)$ and $\pi\left(x_{2}\right)$. Denote this finite collection by $I=\left\{\left[f_{i}\right] \mid i \in I\right\}$. Since $x_{1} \in \pi^{-1}\left(X_{\text {reg }}\right)$, the image $f_{i}\left(\mathbb{P}^{1}\right)$ can only intersect $X_{\text {sing }}$ at finitely many points.

Consider the composition morphism

$$
\mathcal{K}_{0,1}(\mathcal{X}, \beta) \rightarrow \mathcal{K}_{0,1}(X, \beta) \rightarrow \mathcal{K}_{0,0}(X, \beta)
$$

where the morphism $\mathcal{K}_{0,1}(\mathcal{X}, \beta) \rightarrow \mathcal{K}_{0,1}(X, \beta)$ is quasi-finite [2], and the morphism $\mathcal{K}_{0,1}(X, \beta) \rightarrow \mathcal{K}_{0,0}(X, \beta)$ (forgetting the marked point) is projective. Consider any twisted stable map $[f] \in \mathcal{K}_{0,1}(\mathcal{X}, \beta)$. Let $q$ be the stacky point on the domain curve. Since a stacky point can only be mapped to a stacky point, the image $p:=f(q)$ lies in the stacky locus of $\mathcal{X}$ and $\pi(p) \in X_{\text {sing }}$. Note that one can only endow non-trivial stack structures on these points. Therefore, for every $\left[f_{i}\right] \in I$, there are only finitely many ways to endow stack structures on the source curve such that we can lift the stable map (into $X$ ) to a twisted stable map (into $\mathcal{X}$ ). This concludes the proof.

Lemma 3.10. Let $\beta \in H_{2}(X)$ be the homology class in Lemma 3.9. Let $x_{1}$ and $x_{2}$ be two points on $\mathcal{X}$ (not necessarily distinct). Then there are only finitely many stable maps $[h] \in \mathcal{K}_{0,2}(\mathcal{X}, \beta)$ such that $\pi \circ h(0)=\pi\left(x_{1}\right)$ and 
$\pi \circ h(\infty)=\pi\left(x_{2}\right)$, where 0 and $\infty$ are the twisted points on the source curve of $[h]$.

Proof. Consider the forgetful map $\mathcal{K}_{0,2}(\mathcal{X}, \beta) \rightarrow \mathcal{K}_{0,2}(X, \beta)$. Recall that this morphism is quasi-finite and the stack $\mathcal{K}_{0,2}(\mathcal{X}, \beta)$ is proper [2]. Consider the collection of all possible (2-pointed) stable maps $[f] \in \mathcal{K}_{0,2}(X, \beta)$ such that $f(0)=\pi\left(x_{1}\right)$ and $f(\infty)=\pi\left(x_{2}\right)$. It has only finitely many elements by Mori's bend and break. This concludes the proof.

The next proposition is standard:

Proposition 3.11. Assumptions as in Assumptions 3.4. There exists a twisted stable map $f: \mathbb{P}^{1} \rightarrow \mathcal{X}$ such that its $-K_{\mathcal{X}}$-degree is $n+1$ and its image does not meet $\pi^{-1}\left(X_{\text {sing }}\right)$.

Proof. By Proposition 3.7, there is a twisted rational curve $g: \mathbb{P}^{1} \rightarrow \mathcal{X}$ whose image does not lie on $\pi^{-1}\left(X_{\text {sing }}\right)$. Pick a point $q \in g\left(\mathbb{P}^{1}\right) \subset \mathcal{X}$ which is not on $\pi^{-1}\left(X_{\text {sing }}\right)$. The dimension (at the point $[g]$ ) of the family of twisted stable maps $[f] \in \mathcal{K}_{0,0}(\mathcal{X}, n+1)$ whose image contains $q$ and meets $\pi^{-1}\left(X_{\text {sing }}\right)$ is at most $\operatorname{dim} X_{\text {sing }} \leq n-2$ (by a bend and break argument), while the dimension (at $[g])$ of twisted stable maps $[f] \in \mathcal{K}_{0,0}(\mathcal{X}, n+1)$ whose image contains $q$ is at least $n-1$ (by Lemma 2.2 and Claim 3.6). By a simple dimension count, it is easy to see that there is a rational curve which does not meet $\pi^{-1}\left(X_{\text {sing }}\right)$.

Remark 3.12. Let $f: \mathbb{P} \rightarrow \mathcal{X}$ be the twisted stable map (in fact, it is an ordinary stable map) as in Proposition 3.11. Consider an irreducible component containing $[f] \in \mathcal{K}_{0,0}(\mathcal{X}, n+1)$. We can obtain a doubly dominant family of rational curves, i.e. for any general points $x$ and $y$ on $\mathcal{X}$, there is a rational curve in this family through $x$ and $y$.

Notation 3.13. Let $f: \mathbb{P}^{1} \rightarrow \mathcal{X}$ be the rational curve in Proposition 3.7 Let $\beta=\left[(\pi \circ f)_{*}\left(\mathbb{P}^{1}\right)\right] \in R \subset H_{2}(X)$. We will fix this class in the rest of this section.

The next lemma is a simple observation:

Lemma 3.14. Assumptions and notation as in Assumptions 3.4 and Notation 3.13. The Deligne-Mumford stack $\mathcal{K}_{0,0}(\mathcal{X}, \beta)$ is a projective scheme. 
Proof. Let $[f] \in \mathcal{K}_{0,0}(\mathcal{X}, \beta)$. Note that the domain curve is always irreducible since there is no marked point and $n+1$ is the minimal possible $-K_{\mathcal{X}}$-degree. Note that $f: \mathbb{P}^{1} \rightarrow \mathcal{X}$ is birational to its image in $\mathcal{X}$ (again, since $n+1$ is the minimal possible $-K_{\mathcal{X}}$-degree). This implies that there is no non-trivial automorphism for any twisted stable map in $\mathcal{K}_{0,0}(\mathcal{X}, \beta)$. It follows that $\mathcal{K}_{0,0}(\mathcal{X}, \beta)$ is a proper scheme. Since it is quasi-finite to the projective scheme $K_{0,0}(X, \beta)$. It has to be projective.

Let $[f] \in \mathcal{K}_{0,0}(\mathcal{X}, \beta)$ be a twisted stable map into $\mathcal{X}$ which does not meet the preimage of $X_{\text {sing }}$. Let $Z \subset \mathcal{K}_{0,0}(X, \beta)$ be an irreducible component which contains $[f]$ and $\tilde{Z}$ the normalization. Consider the finite morphism $\mathcal{K}_{0,0}(\mathcal{X}, \beta) \rightarrow K_{0,0}(X, \beta)$. Let $Z^{\prime}$ be the component of $K_{0,0}(X, \beta)$ which contains the image of $Z$, i.e. stable maps of the form $[\pi \circ h]$ with $[h] \in Z$. We also take the normalization $\tilde{Z}^{\prime}$ of $Z^{\prime}$. The next lemma compares these two components.

Lemma 3.15 ([5] Lemma 3.12). The natural map $\tilde{Z} \rightarrow \tilde{Z}^{\prime}$ is an isomorphism.

\section{Proof of Theorem 1.2}

We give a quick proof of Theorem 1.2 in this section. Return to work on the variety $X$, rather than the stack $\mathcal{X}$. Recall the main theorem from [4]:

Theorem 4.1 (=4] Main Theorem 0.1). Let $X$ be a normal projective variety over $\mathbb{C}$. If $X$ carries a closed, maximal, unsplitting doubly dominant family of rational curves, then $X$ is isomorphic to $\mathbb{P}^{n}$.

The existence of such a family of rational curves follows from Proposition 3.11 and Remark 3.12. This concludes the proof of Theorem 1.2 .

Remark 4.2. There is a more elementary proof based on the argument from [10]. The reader can consult [5] Section 4 for more details.

Remark 4.3. If $X$ has at worst LCIQ singularities, we can prove the same result with an extra assumption that $\operatorname{dim} X_{\text {sing }}<(n-1) / 2$. H.-H. Tseng and I are working on removing this extra assumption. 


\section{References}

1. D. Abramovich, A. Corti, and A. Vistoli, Twisted bundles and admissible covers, Comm. Algebra, 31 (2003), 3547-3618.

2. D. Abramovich and A. Vistoli. Compactifying the space of stable maps, J. Amer. Math. Soc., 15 (2002), no. 1, 27-75.

3. F. Ambro, Quasi-log varieties, Tr. Mat. Inst. Steklova, 240 (2003), Biratsion. Geom. Linein. Sist. Konechno Porozhdennye Algebry, 220-239; translation in Proc. Steklov Inst. Math., 240 (2003), no. 1, 214-233.

4. K. Cho, Y. Miyaoka and N. I. Shepherd-Barron. Characterizations of projective spaces and applications to complex symplectic manifolds, Higher dimensional birational geometry (Kyoto, 1997), 1-88, Adv. Stud. Pure Math., 35, Math. Soc. Japan, Tokyo, 2002.

5. J.- C. Chen and H.- H. Tseng. Note on Characterization of Projective spaces, Comm. in Algebra, 35 (2007), Issue 11, 3808-3819.

6. J.- C. Chen and H.- H. Tseng. Cone Theorem via Deligne-Mumford stacks, Mathematische Annalen, 345 (2009), no. 3, 525-545.

7. O. Debarre. Higher-dimensional Algebraic Geometry, Springer-Verlag, 2001.

8. Y. Kawamata, On the length of an extremal rational curve, Invent. Math., 105 (1991), no. 3, 609-611.

9. S. Kebekus. Families of singular rational curves, J. Algebraic Geom., 11 (2002), no. 2, 245-256, math.AG/0004023.

10. S. Kebekus. Characterizing the projective space after Cho, Miyaoka and ShepherdBarron, Complex Geometry (Göttingen, 2000), 147-155, Springer, Berlin, 2002, math.AG/0107069.

11. J. Kollár. Rational Curves on Algebraic Varieties, Springer-Verlag, 1996.

12. J. Kollár, S. Mori. Birational Geometry of Algebraic Varieties, Cambridge Tracts in Mathematics 134, Cambridge University Press, 1998. 